\title{
More evidence cannabis can help in neuropathic pain
}

\author{
Henry J. McQuay DM \\ Previously published at www.cmaj.ca \\ $\infty \quad$ See related research article by Ware and colleagues, page E694
}

$\mathrm{I}$ t's good to see the trial of smoked cannabis in neuropathic pain reported by Ware and colleagues ${ }^{1}$ because smoking is the most common way in which patients try this drug. The authors should be congratulated for tackling the question of whether cannabis helps in neuropathic pain, particularly given that the regulatory hurdles for their trial must have been a nightmare. The question is worth investigating because of the ongoing publicity - which patients see, hear and read - that suggests an analgesic activity of cannabis in neuropathic pain, and because of the paucity of robust evidence for such an analgesic effect. If patients are not achieving a good response with conventional treatment of their pain, then they may, reasonably, wish to try cannabis. If medical cannabis is not available where a patient lives, then obtaining it will take the patient outside of the law, often for the first time in his or her life. Good evidence would at least buttress that decision.

This trial adds to the three previous studies of smoked cannabis in neuropathic pain that I could find using PubMed and reference lists. Two of these studies were restricted to neuropathic pain in patients with HIV., ${ }^{2,3}$ Wilsey and colleagues studied patients with peripheral or central neuropathic pain, whereas Ware and colleagues" studied people with "neuropathic pain of at least three months duration due to trauma or surgery, with allodynia or hyperalgesia."

The crossover trial by Ware and colleagues ${ }^{1}$ was small, with only 21 participants completing the trial, and the authors were unable to recruit the numbers planned. Other than that limitation, the trial checks off all of the requisite boxes for appropriate design and execution. The authors state that their trial of smoked cannabis is noteworthy because it involved outpatients who took multiple doses, whereas other studies have been conducted in residential "laboratories," with patients taking just one dose at each time point. The trial did not last long (i.e., three doses were given on each of five days in each of four treatment arms), so the authors cannot really say whether any response would be sustained. Furthermore, the results are presented as averages. The average daily pain intensity was significantly lower, but not hugely so, for patients taking $9.4 \%$ tetrohydrocannabinol relative to those receiving $0 \%$ tetrohydrocannabinol. This finding, in this patient group, contrasts with the poor outcome obtained for use of oral cannabis relative to dihydrocodeine reported by Frank and colleagues. $^{5}$

\section{Key points}

- Current treatments do not help all patients with neuropathic pain.

- Cannabis can produce moderate analgesia in patients with neuropathic pain.

- This analgesic effect may be more pronounced in central, as opposed to peripheral, neuropathic pain.

Details about patients with and without a response to treatment would have been helpful. In particular, do specific pain mechanisms respond to cannabis and others not? Whether valid predictors of success exist for treatment with cannabis would be useful for patients and prescribers to know. This deficit in the study emphasizes the general point that analysis of treatment responders is important for getting the most out of unique sets of data. Adverse events were well studied in this trial. No clear dose-response relation was evident for the expected effects of cannabis on the central nervous system. However, this finding may reflect the fact that $80 \%$ of participants reported having had prior exposure to the drug (albeit not recent or substantial exposure).

Putting together the four trials of smoked cannabis, the provisional conclusions are that an analgesic effect is evident, that this effect, though not great, may be of use to some patients, and that it often carries with it some adverse effects on the central nervous system (though not obviously so in this trial). These conclusions make biological sense, given that cannabinoids taken orally have shown the same sorts of effects. ${ }^{6}$ Interestingly, the "moderate" analgesic effect shown here for neuropathic pain seems to hold true for nociceptive pain.

Few drugs show efficacy for both nociceptive and neuropathic pain. Ketamine is one. Opioids do it too in some forms of neuropathic pain, but with a substantial shift to the right on the dose-response curve. Whether cannabinoids can reduce both nociceptive and neuropathic pain at equivalent doses (thus avoiding increased adverse effects because higher doses would not be needed) will be interesting to see. Whether they show greater efficacy in central than in peripheral neuropathic pain (given that current treatments work less well for central pain) will also be interesting to see.

Henry J. McQuay is an Emeritus Fellow at Balliol College, Oxford, UK.

CMAJ 2010. DOI:10.1503/cmaj.100799

All editorial matter in CMAJ represents the opinions of the authors and not necessarily those of the Canadian Medical Association. 
In some forms of central neuropathic pain (e.g., pain associated with multiple sclerosis), the distinguishing characteristics of that which is truly nociceptive and that which is neuropathic are blurred. Commonly, such patients take conventional analgesics for their nociceptive pain as well as unconventional analgesics, antidepressants and antiepileptics for the neuropathic element of their pain. The two small trials of smoked cannabis for HIV-related pain are important because little else works well for this type of pain. On the basis of these two trials, Attal and colleagues, ${ }^{8}$ writing for the European Federation of Neurological Societies, endorsed cannabinoids for second-line use in the treatment of central neuropathic pain. This conclusion seems sensible, and is supported by positive results from other randomized trials (e.g., a study by Svendsen and colleagues ${ }^{6}$ involving patients with multiple sclerosis).

Whether drugs capable of exploiting the differences between the various cannabinoid receptors can yield greater analgesia with fewer adverse effects remains to be seen. This capability would be of substantial benefit in the treatment of neuropathic pain, particularly that of central origin, because existing treatments are far from perfect. In the meantime, the current trial adds to the trickle of evidence that cannabis may help some of the patients who are struggling at present.
Competing interests: Dr. McQuay has been a member of advisory boards for Reckitt Benckiser, Pfizer Data Safety and Monitoring Board and Archimedes, and has been a consultant to Esteve, Sanofi and Archimedes. He has provided expert testimony supported by Ratiopharm and Sandoz. He has received payment for lectures from Archimedes and Grunenthal, and has received royalties for a textbook on the subject of pain.

\section{REFERENCES}

1. Ware MA, Wang T, Shapiro S, et al. Smoked cannabis for chronic neuropathic pain: a randomized controlled trial. CMAJ 2010. DOI:10.1503/cmaj.091414.

2. Ellis RJ, Toperoff W, Vaida F, et al. Smoked medicinal cannabis for neuropathic pain in HIV: a randomized, crossover clinical trial. Neuropsychopharmacology 2009;34:672-80.

3. Abrams DI, Jay CA, Shade SB, et al. Cannabis in painful HIV-associated sensory neuropathy: a randomized placebo-controlled trial. Neurology 2007;68:515-21.

4. Wilsey B, Marcotte T, Tsodikov A, et al. A randomized, placebo-controlled, crossover trial of cannabis cigarettes in neuropathic pain. J Pain 2008;9:506-21.

5. Frank B, Serpell MG, Hughes J, et al. Comparison of analgesic effects and patient tolerability of nabilone and dihydrocodeine for chronic neuropathic pain: randomised, crossover, double blind study. BMJ 2008;336:199-201.

6. Svendsen KB, Jensen TS, Bach FW. Does the cannabinoid dronabinol reduce central pain in multiple sclerosis? Randomised double blind placebo controlled crossover trial. BMJ 2004;329:253.

7. Campbell FA, Tramer MR, Carroll D, et al. Are cannabinoids an effective and safe treatment option in the management of pain? A qualitative systematic review. BMJ 2001;323:1-6

8. Attal N, Cruccu G, Baron R, et al. EFNS guidelines on the pharmacological treatment of neuropathic pain: 2010 revision. Eur J Neurol 2010;17:1113-e88.

Correspondence to: Henry J. McQuay, 16 Stansfield Close,

Oxford OX38THUK; henrymcquay@gmail.com

\section{PrACGUPRIL ${ }^{\circledR}$ and PAGCURETIG ${ }^{\circledR}$} Still fighting hypertensionl

$\checkmark$ Powerful BP-lowering ${ }^{1}$

$\checkmark$ ACCUPRIL's 24-hour BP control ${ }^{1}$

$\checkmark$ The flexibility of seven dosage strengths with ACCUPRIL and ACCURETIC ${ }^{1,2}$

$\checkmark$ Al dosages at a single flat price ${ }^{3 \dagger}$

† Price does not include pharmacy professional fees. Please refer to Product Monographs for complete dosing information.

P

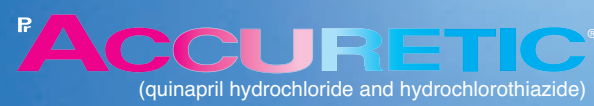

POWER OF COMBINED CONTROL

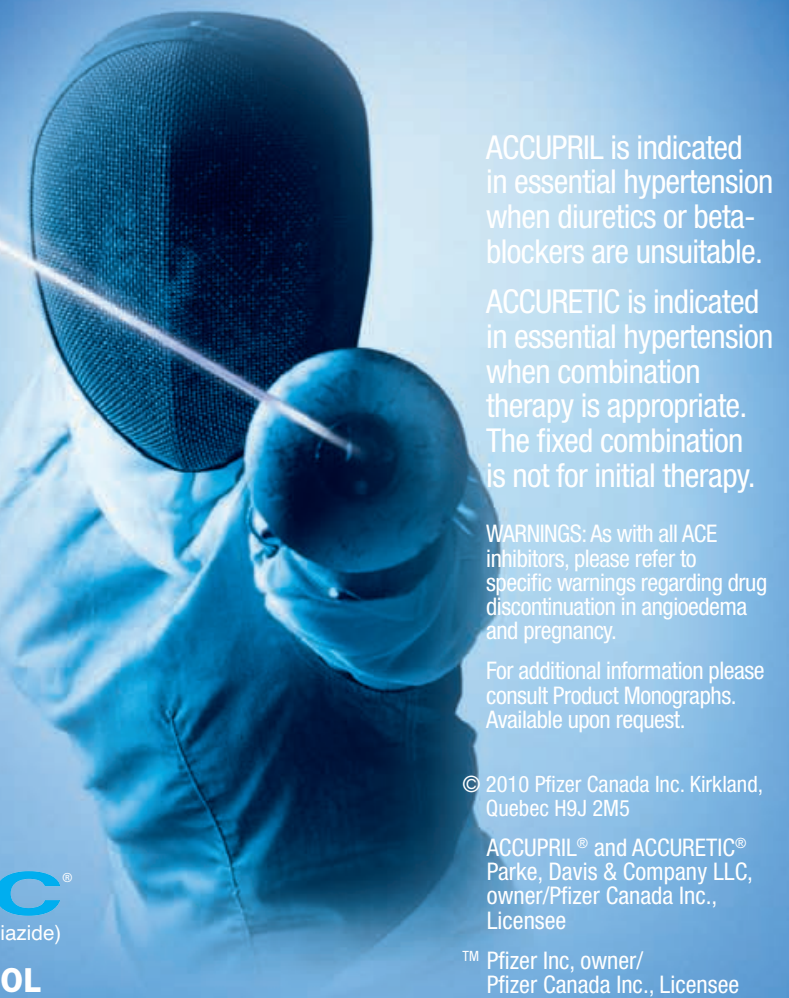

